\title{
Temperature effects during Ostwald ripening
}

\author{
Giridhar Madras* \\ Department of Chemical Engineering, Indian Institute of Science, Bangalore 560 012, India
}

Benjamin J. McCoy

Department of Chemical Engineering, Louisiana State University, Baton Rouge, Louisiana 70803

\begin{abstract}
Temperature influences Ostwald ripening through its effect on interfacial energy, growth rate coefficients, and equilibrium solubility. We have applied a distribution kinetics model to examine such temperature effects. The model accounts for the Gibbs-Thomson influence that favors growth of larger particles, and the dissolution of unstable particles smaller than critical nucleus size. Scaled equations for the particle size distribution and solution concentration as functions of time are solved numerically. Moments of the distribution show the temporal evolution of number and mass concentration, average particle size, and polydispersity index. Parametric and asymptotic trends are plotted and discussed in relation to reported observations. Temperature programming is proposed as a potential method to control the size distribution during the phase transition. We also explore how two crystal polymorphs can be separated by a temperature program based on different interfacial properties of the crystal forms.
\end{abstract}

\section{INTRODUCTION}

Particle growth during phase transitions in materials and pharmaceutical processing is influenced by kinetics and thermodynamics through temperature effects. The effect of temperature on interfacial energy, diffusion and growth rate coefficients, and equilibrium solubility at the microstructural level influences crystal or grain properties during the phase transition. Thus temperature is a potential control parameter that can be manipulated to optimize product properties and manufacturing methods. Ostwald ripening is the last stage of a condensation transition from gas to liquid or from liquid to solid. ${ }^{1-3}$ During ripening of a distribution of particles, the Gibbs-Thomson effect determines that smaller particles are more soluble than larger particles. ${ }^{4}$ Smaller particles can shrink to their critical nucleus size and rapidly vanish because of the thermodynamic instability of subcritical clusters. ${ }^{5}$ This denucleation process leads to a diminution in the number of particles, and a consequent asymptotic powerlaw evolution to a monodisperse distribution, ultimately consisting of a single large particle. ${ }^{6}$ All the participating processes are affected by the temperature as the system proceeds toward its asymptotic behavior. Our aim is to explore the possibility that temperature programming can provide a way to tailor the particle distribution during ripening.

Among the earliest models for the particle size distribution were those of Lifschitz and Slyozov ${ }^{7}$ (LS) and Wagner ${ }^{8}$ (W), whose approximations included assuming the monomer concentration is constant at its equilibrium value. Marqusee and Ross ${ }^{9}$ expanded on the LSW model by showing it represents the leading terms in a series for the long time solution. Venzl ${ }^{10}$ solved the governing first-order nonlinear dif-

*Author to whom correspondence should be addressed. Tel.: 91-080-309-2321; Fax: 91-080-360-0683; Electronic mail: giridhar@chemeng.iisc.ernet.in ferential equation numerically, assuming that clusters vanished at a rate varying exponentially or inversely with time. Bhakta and Ruckenstein ${ }^{11}$ more recently based a stochastic theory of ripening on a discrete microscopic continuity equation that generalized the LSW differential equation with rate constants assumed independent of particle size. We have recently ${ }^{4-6}$ formulated a new approach to Ostwald ripening (or isothermal recrystallization) that accounts for the evolution of the particle size distribution expressed in terms of the particle mass, rather than its radius. The distributionkinetics approach with single monomer addition and dissociation is reversible and is generally applicable to growth, dissolution, or ripening phenomena. Denucleation of unstable clusters ensures that the cluster number decreases as required for a realistic model of ripening. We have shown ${ }^{12}$ how the LSW model ${ }^{7,8}$ and subsequent enhancements of the model by other investigators ${ }^{9,10,13-17}$ correctly depict the time dependence of particle number concentration and average particle size, but often approximate the higher moments of the particle size distribution.

Particles with more than one crystal structure, ${ }^{18}$ or polymorphs, provide an example for investigation. Polymorphs with different shapes have different surface properties, which influences the growth rate of crystal faces and shape the crystal habit. ${ }^{19}$ Two polymorphs affected differently by temperature will respond differently to temperature varying with time. One polymorph may be more stable at a given temperature than another, and thus the more stable form would grow faster whereas the less stable form would grow slower. Controlling the particle size distributions can potentially be optimized by applying a temperature program.

Our theory ignores temperature gradients within the melt-particle system, which should be valid when the Prandtl number is small $(\operatorname{Pr} \ll 1)$. Heat conduction in the presence of an imposed linear temperature gradient has been considered 
by Snyder et al. ${ }^{20}$ in a numerical simulation of particle coarsening.

The temperature effects incorporated into the present model include the diffusion-influenced growth coefficient, the Gibbs-Thomson effect of particle curvature on equilibrium solubility, the phase-transition energy (heat of solidification or vaporization), the critical nucleus size, and interfacial energy (surface tension). The dissolution rate coefficient is related to the growth rate coefficient by microscopic reversibility, thereby determining its temperature dependence. The absolute temperature is scaled by a reference temperature, which for gas-liquid systems is the critical temperature $T_{c}$.

In an earlier paper $^{21}$ we presented a crystal growth theory with temperature effects, whereas in the present work we focus on Ostwald ripening. Ripening is caused by the varying curvature of different interfaces, and thus can be important whenever a distribution of particle sizes exists. The consequent interfacial energy (or Gibbs-Thomson) effect is sensitive to temperature, thus offering an opportunity to control cluster or grain size by temperature programming. For two polymorphs, any of the kinetic or thermodynamic parameters in the model might have different values, but the effect of the interfacial energy coefficient is a property of particular interest. In what follows, we examine the temperature dependence of the parameters that influence ripening. We begin by discussing the elements of distribution kinetics through population dynamics (Sec. II), then propose an asymptotic solution to the dimensionless population dynamics equation (Sec. III), present and discuss the results of the numerical analysis of the population dynamics equation (Sec. IV), and finally provide comparisons with experimental observations along with conclusions (Sec. V).

\section{DISTRIBUTION KINETICS}

The size distribution is defined by $c(x, t) d x$, representing the concentration of clusters (crystals, droplets, particles) at time $t$ in the differential mass range $(x, x+d x)$. Moments are defined as integrals over the mass,

$$
c^{(n)}(t)=\int_{0}^{\infty} c(x, t) x^{n} d x .
$$

The zeroth moment, $c^{(0)}(t)$, and the first moment, $c^{(1)}(t)$, are the time-dependent molar (or number) concentration of clusters and the cluster mass concentration (mass/volume), respectively. The ratio of the two is the average cluster mass, $c^{\text {avg }}=c^{(1)} / c^{(0)}$. The variance, $c^{\mathrm{var}}=c^{(2)} / c^{(0)}-\left[c^{\mathrm{avg}}\right]^{2}$, and the polydispersity index, $c^{\mathrm{pd}}=c^{(2)} c^{(0)} / c^{(1) 2}$, are measures of the polydispersity. The molar concentration, $m^{(0)}(t)$, of solute monomer of molecular weight $x_{m}$ is the zeroth moment of the monomer distribution, $m(x, t)=m^{(0)}(t) \delta\left(x-x_{m}\right)$.

The deposition or condensation process by which monomers of mass $x^{\prime}=x_{m}$ are reversibly added to or dissociated from a cluster of mass $x$ can be written as the reactionlike process, ${ }^{22,23}$

$$
\mathbf{C}(x)+\mathbf{M}\left(x^{\prime}\right) \underset{k_{g}(x)}{\stackrel{k_{d}(x)}{\rightleftarrows}} \mathbf{C}\left(x+x^{\prime}\right),
$$

where $\mathbf{C}(x)$ is the cluster of mass $x$ and $\mathbf{M}\left(x^{\prime}=x_{m}\right)$ is the monomer. The mass balance equations governing the cluster distribution, $c(x, t)$, and the monomer distribution, $m(x, t)$, are

$$
\begin{aligned}
\partial c(x, t) / \partial t= & -k_{g}(x) c(x, t) \int_{0}^{\infty} m\left(x^{\prime}, t\right) d x^{\prime} \\
& +\int_{0}^{x} k_{g}\left(x-x^{\prime}\right) c\left(x-x^{\prime}, t\right) m\left(x^{\prime}, t\right) d x^{\prime} \\
& -k_{d}(x) c(x, t)+\int_{x}^{\infty} k_{d}\left(x^{\prime}\right) c\left(x^{\prime}, t\right) \\
& \times \delta\left(x-\left(x^{\prime}-x_{m}\right)\right) d x^{\prime}-I \delta\left(x-x^{*}\right)
\end{aligned}
$$

and

$$
\begin{aligned}
\partial m(x, t) / \partial t= & -m(x, t) \int_{0}^{\infty} k_{g}\left(x^{\prime}\right) c\left(x^{\prime}, t\right) d x^{\prime} \\
& +\int_{x}^{\infty} k_{d}\left(x^{\prime}\right) c\left(x^{\prime}, t\right) \delta\left(x-x_{m}\right) d x^{\prime} \\
& +I \delta\left(x-x^{*}\right) x^{*} / x_{m} .
\end{aligned}
$$

Nucleation of clusters of mass $x^{*}$ at rate $I$ are source terms or, in case of ripening, sink terms for denucleation, which occurs when clusters shrink to their critical size, $x^{*}$, and then spontaneously vanish. The difference between ordinary dissolution due to concentration driving forces and total disintegration due to thermodynamic instability is thus underscored. This is the key distinction between crystal growth ${ }^{21}$ alone and the present discussion of growth with ripening. For ordinary particle growth or dissolution, we would set $I=0$. Initial conditions for Eqs. (2.3) and (2.4) are $c(x, t=0)$ $=c_{0}(x)$ and $m(x, t=0)=m_{0}^{(0)} \delta\left(x-x_{m}\right)$. The mass balance follows from Eqs. (2.3)-(2.4), and can be expressed in terms of mass concentrations,

$$
x_{m} m_{0}^{(0)}+c_{0}^{(1)}=x_{m} m^{(0)}(t)+c^{(1)}(t) .
$$

The size distribution changes according to Eq. (2.3), which becomes, when the integrations over the Dirac distributions are performed, the finite-difference differential equation,

$$
\begin{aligned}
\partial c(x, t) / \partial t= & -k_{g}(x) c(x, t) m^{(0)}(t) \\
& +k_{g}\left(x-x_{m} c\left(x-x_{m}, t\right) m^{(0)}(t)\right. \\
& -k_{d}(x) c(x, t)+k_{d}\left(x+x_{m}\right) c\left(x+x_{m}, t\right) \\
& -I \delta\left(x-x^{*}\right) .
\end{aligned}
$$

Equation (2.5) can be expanded for $x_{m} \ll x$ to convert the differences into differentials, yielding the customary (approximate) continuity equation applied to particle growth and ripening. ${ }^{4,6,15}$

At equilibrium, $\partial c / \partial t=0$ and $I=0$, so that Eq. (2.5) implies

$$
k_{d}(x)=m_{\mathrm{eq}}^{(0)} k_{g}(x),
$$


which is a statement of microscopic reversibility (detailed balance). With rate coefficients for a cluster of mass, $x$, given an expression for $k_{g}(x)$, one can calculate $k_{d}(x)$.

A monomer that attaches to a cluster may diffuse through the solution to react at the cluster surface. Such diffusion-controlled reactions have a rate coefficient represented ${ }^{24}$ by

$$
k_{g}=4 \pi D_{m} r_{c},
$$

where the cluster radius is related to its mass $x$ by $r_{c}$ $=\left(3 x / 4 \pi \rho_{c}\right)^{1 / 3}$, in terms of the cluster mass density $\rho_{c}$, which we assume to be constant with temperature. As usual in kinetics, the temperature dependence of the growth (addition, aggregation) rate is quite weak relative to dissolution (dissociation, scission) rate. For the large range of temperatures in some ceramic processing methods, however, diffusion effects can be significant, so we assume an activation energy for the growth coefficient to account for activated diffusion,

$$
D_{m}=D_{0} \exp (-E / R T)
$$

thus

$$
k_{g}(x)=\gamma x^{\lambda} \exp (-E / R T),
$$

where $E$ is the activation energy, $R$ is the gas constant, and if $\lambda=1 / 3$, then $\gamma=4 \pi D_{0}\left(3 / 4 \pi \rho_{c}\right)^{1 / 3}$. The $1 / 3$ power on $x$ thus represents diffusion-controlled ripening, the primary issue in previous work. ${ }^{13-15}$ When growth is limited by monomer attachment and dissociation at the cluster surface, the rate coefficient is proportional to the cluster surface area, $k_{g}$ $\propto r_{c}^{2}$, so that we can write $k_{g}$ proportional to $x^{2 / 3}$; thus in Eq. (2.8), $\lambda=2 / 3$ for surface-controlled ripening. ${ }^{6}$ If the deposition is independent of the surface area, then $k_{g}$ varies as $x^{0}$. Other expressions for the rate coefficients that are applicable to cluster growth may be realistic for complex and combined rate processes.

The temperature dependence for growth and ripening is influenced by the thermodynamic properties. The interfacial curvature effect is prescribed by the Gibbs-Thomson equation expressed in terms of $m_{\infty}^{(0)}$, the equilibrium solubility (or vapor pressure) of a plane surface,

$$
m_{\mathrm{eq}}^{(0)}=m_{\infty}^{(0)} \exp (\Omega)
$$

with

$$
\Omega=2 \sigma x_{m} / r_{c} \rho_{c} k_{B} T,
$$

where $x_{m} / \rho_{c}$ is monomer molar volume, $\sigma$ is the interfacial energy, $k_{B}$ is Boltzmann's constant, and $T$ is temperature. Asymptotic models ${ }^{1,14}$ always linearize the Gibbs-Thomson equation, an approximation not valid for small particles during early stages of ripening or at low temperatures ${ }^{25}$ according to Eq. (2.10).

The critical nucleus radius at a given solute concentration $m^{(0)}$ is

$$
r^{*}=2 \sigma x_{m} /\left[\rho_{c} k_{B} T \ln \left(m^{(0)} / m_{\infty}^{(0)}\right)\right] .
$$

Surface tension for the gas-liquid interface decreases nearly linearly with temperature, ${ }^{26}$ thus we take $\sigma=\sigma_{0}\left(1-T / T_{c}\right)$, where $T_{c}$ is the critical (or reference) temperature, causing $\sigma$ to vanish at the critical point. For liquid-solid interfaces, the temperature dependence of $\sigma$ may be represented by a more complex function. The temperature dependence of the equilibrium solubility is given by

$$
m_{\infty}^{(0)}=\mu_{\infty} \exp (-\Delta H / R T),
$$

where $\Delta H$ is the molar energy of the phase transition, and $\mu_{\infty}$ is the flat-surface equilibrium solubility at large $T$.

We define scaled dimensionless quantities for the mass and temperature relationships,

$$
\begin{aligned}
& C=c x_{m} / \mu_{\infty}, \quad C^{(n)}=c^{(n)} / \mu_{\infty} x_{m}^{n}, \quad \xi=x / x_{m}, \\
& \theta=t \gamma \mu_{\infty} x_{m}^{\lambda}, \quad S=m^{(0)} / \mu_{\infty}, \\
& S_{\mathrm{eq}}=S \exp (h / \Theta-\Omega), \quad \Omega=w\left(\Theta^{-1}-1\right) \xi^{1 / 3}, \\
& w=\left(3 x_{m} / 4 \pi \rho_{c}\right)^{-1 / 3} 2 \sigma_{0} x_{m} / \rho_{c} k_{B} T_{c}, \\
& \Theta=T / T_{c}, \quad J=I /\left(\gamma \mu_{\infty}^{2} x_{m}^{\lambda}\right), \quad h=\Delta H / R T_{c}, \\
& \epsilon=E / R T_{c} .
\end{aligned}
$$

Note that $\xi$ is the number of monomers in the cluster and $\Theta$ is the reduced temperature $(0<\Theta<1)$. The ratio $S$ is defined relative to the high temperature solubility $\mu_{\infty}$, rather than to the plane-surface solubility $m_{\infty}^{(0)}$ as in our earlier isothermal work. ${ }^{4,6}$ The supersaturation ratio defined as $S_{\mathrm{eq}}=m^{(0)} / m_{\mathrm{eq}}^{(0)}$ evolves to unity at thermodynamic equilibrium. The scaled number (or moles) of particles, $C^{(0)}=c^{(0)} / \mu_{\infty}$, is also in units of the solubility $\mu_{\infty}$. The Gibbs-Thomson factor $\Omega$, Eq. (2.13), is expressed in terms of a scaled interfacial energy, $w$. Substituting these expressions in Eqs. (2.3) and (2.4) yields the dimensionless equations,

$$
\begin{aligned}
\partial C(\xi, \theta) / \partial \theta= & S(\theta) \exp (-\epsilon / \Theta)\left[-\xi^{\lambda} C(\xi, \theta)\right. \\
& \left.+(\xi-1)^{\lambda} C(\xi-1, \theta)\right]-\xi^{\lambda} \exp [-(h \\
& +\epsilon) / \Theta] \exp \left[w\left(\Theta^{-1}-1\right) \xi^{-1 / 3}\right] C(\xi, \theta) \\
& +(\xi+1)^{\lambda} \exp [-(h+\epsilon) / \Theta] \exp \left[w \left(\Theta^{-1}\right.\right. \\
& \left.-1)(\xi+1)^{-1 / 3}\right] C(\xi+1, \theta)-J \delta\left(\xi-\xi^{*}\right)
\end{aligned}
$$

and

$$
\begin{aligned}
d S(\theta) / d \theta= & \exp (-\epsilon / \Theta)[-S(\theta)+\exp (-h / \Theta) \\
& \left.\times \exp \left[w\left(\Theta^{-1}-1\right)\left(C^{\mathrm{avg}}\right)^{-1 / 3}\right]\right] C^{(\lambda)}+J \xi^{*} .
\end{aligned}
$$

The initial conditions are $S(\theta=0)=S_{0}$ and $C(\xi, \theta=0)$ $=C_{0}(\xi)$. Because the rate coefficients are related by microscopic reversibility in Eq. (2.4), Eq. (2.15) provides the required thermodynamic equilibrium, $m^{(0)}=m_{\mathrm{eq}}^{(0)}$, when $d S / d \theta=0$ and $J=0$. The number of monomers in the critical nucleus is

$$
\xi^{*}=\left[w\left(\Theta^{-1}-1\right) /(\ln S+h / \Theta)\right]^{3},
$$

which varies with time because of the time dependence of $\Theta(\theta)$ and $S(\theta)$.

From Eq. (2.1) the scaled moments are 


$$
C^{(n)}(\theta)=\int_{0}^{\infty} C(\xi, \theta) \xi^{n} d \xi .
$$

The scaled mass balance for a closed (batch) system follows from Eq. (2.4a),

$$
C^{(1)}(\theta)+S(\theta)=C_{0}^{(1)}+S_{0} .
$$

Polymorphs: For A and B polymorphs we assume that differences are determined by the interfacial energies, $w_{\mathrm{A}}$ and $w_{\mathrm{B}}$, rather than activation or phase transition energies. Thus, the interfacial coefficients $\sigma_{\mathrm{A} 0}$ and $\sigma_{\mathrm{B} 0}$ are the only terms in the definitions that affect $w_{\mathrm{A}}$ and $w_{\mathrm{B}}$. With appropriate subscripts we distinguish between the two distributions, $C_{\mathrm{A}}(\xi, \theta)$ and $C_{\mathrm{B}}(\xi, \theta)$, which have moments defined as in Eq. (2.17). The same solute produces the two polymorphs, so the mass balance is

$$
C_{\mathrm{A}}^{(1)}(\theta)+C_{\mathrm{B}}^{(1)}(\theta)+S(\theta)=C_{\mathrm{A} 0}^{(1)}+C_{\mathrm{B} 0}^{(1)}+S_{0} .
$$

The governing population balance equations are written,

$$
\begin{aligned}
\partial C_{\mathrm{A}}(\xi, \theta) / \partial \theta= & S(\theta) \exp (-\epsilon / \Theta)\left[-\xi^{\lambda} C_{\mathrm{A}}(\xi, \theta)\right. \\
& \left.+(\xi-1)^{\lambda} C_{\mathrm{A}}(\xi-1, \theta)\right]-\xi^{\lambda} \\
& \times \exp [-(h+\epsilon) / \Theta] \exp \left[w _ { \mathrm { A } } \left(\Theta^{-1}\right.\right. \\
& \left.-1) \xi^{-1 / 3}\right] C_{\mathrm{A}}(\xi, \theta)+(\xi+1)^{\lambda} \\
& \times \exp [-(h+\epsilon) / \Theta] \exp \left[w_{\mathrm{A}}\left(\Theta^{-1}-1\right)\right. \\
& \left.\times(\xi+1)^{-1 / 3}\right] C_{\mathrm{A}}(\xi+1, \theta)-J_{\mathrm{A}} \delta\left(\xi-\xi_{\mathrm{A}}^{*}\right)
\end{aligned}
$$

and

$$
\begin{aligned}
\partial C_{\mathrm{B}}(\xi, \theta) / \partial \theta= & S(\theta) \exp (-\epsilon / \Theta)\left[-\xi^{\lambda} C_{\mathrm{B}}(\xi, \theta)\right. \\
& \left.+(\xi-1)^{\lambda} C_{\mathrm{B}}(\xi-1, \theta)\right]-\xi^{\lambda} \\
& \times \exp [-(h+\epsilon) / \Theta] \exp \left[w_{\mathrm{B}}\left(\Theta^{-1}-1\right) \xi^{-1 / 3}\right] \\
& \times C_{\mathrm{B}}(\xi, \theta)+(\xi+1)^{\lambda} \\
& \times \exp [-(h+\epsilon) / \Theta] \exp \left[w_{\mathrm{B}}\left(\Theta^{-1}-1\right)\right. \\
& \left.\times(\xi+1)^{-1 / 3}\right] C_{\mathrm{B}}(\xi+1, \theta)-J_{\mathrm{B}} \delta\left(\xi-\xi_{\mathrm{B}}^{*}\right)
\end{aligned}
$$

with

$$
\begin{aligned}
d S(\theta) / d \theta= & -S(\theta) \exp (-\epsilon / \Theta)+\exp (-\epsilon / \Theta) \\
& \times\left\{\left[\exp (-h / \Theta) \exp \left[w_{\mathrm{A}}\left(\Theta^{-1}-1\right)\left(C_{\mathrm{A}}^{\mathrm{avg}}\right)^{-1 / 3}\right]\right]\right. \\
& +C_{\mathrm{B}}^{(\lambda)}+\left[\operatorname { e x p } ( - h / \Theta ) \operatorname { e x p } \left[w_{\mathrm{B}}\left(\Theta^{-1}-1\right)\right.\right. \\
& \left.\left.\left.\times\left(C_{\mathrm{B}}^{\mathrm{avg}}\right)^{-1 / 3}\right]\right] C_{\mathrm{B}}^{(\lambda)}\right\}+J_{\mathrm{A}} \xi_{\mathrm{A}}^{*}+J_{\mathrm{B}} \xi_{\mathrm{B}}^{*} .
\end{aligned}
$$

The initial conditions are $S(\theta=0)=S_{0}, \quad C_{\mathrm{A}}(\xi, \theta=0)$ $=C_{\mathrm{A} 0}(\xi)$, and $C_{\mathrm{B}}(\xi, \theta=0)=C_{\mathrm{B} 0}(\xi)$. The terms $J_{\mathrm{A}} \xi_{\mathrm{A}}^{*}$ $+J_{\mathrm{B}} \xi_{\mathrm{B}}^{*}$ in Eq. (2.22) account for the mass added to the solution as polymorphs $\mathrm{A}$ and $\mathrm{B}$ denucleate.

\section{ASYMPTOTIC SOLUTION}

A long-time asymptotic solution that shows the temperature effect can be constructed along the lines of our earlier work. ${ }^{6}$ The PBE, Eq. (2.14), can be converted into a FokkerPlanck equation by expanding $C(\xi \pm 1, \theta)$ around $\xi=1$ and keeping first-order terms,

$$
\begin{aligned}
\partial C(\xi, \theta) / \partial \theta= & \exp [-h+\epsilon) / \Theta] \partial\left[\xi^{\lambda}\{S \exp (h / \Theta)\right. \\
& \left.\left.-\exp \left[w(1 / \Theta-1) / \xi^{1 / 3}\right]\right\} C(\xi, \theta)\right] / \partial \xi \\
& -J \delta\left(\xi-\xi^{*}\right) .
\end{aligned}
$$

The asymptotic solution requires that $S_{\text {eq }}=S \exp (h / \Theta) \rightarrow 1$, so an expansion of the terms in brackets, \{\} , yields

$$
S \exp (h / \Theta)-\exp \left[w(1 / \Theta-1) / \xi^{1 / 3}\right] \sim w(1 / \Theta-1) / \xi^{1 / 3} .
$$

Because the number of monomers in a particle grows with time, $\xi^{-1 / 3}$ will eventually become small enough to justify keeping only one term in the expansion [Eq. (3.2)].

The moment equations for Eqs. (3.1) and (3.2) are found by multiplying by $\xi^{n}$ and integrating (the second term by parts) according to the moment definition, Eq. (2.17),

$$
\begin{aligned}
d C^{(n)} / d \theta= & -n \exp [-(h+\epsilon) / \Theta] w(1 / \Theta-1) \\
& \times C^{(n+\lambda-4 / 3)}-J \xi^{* n}
\end{aligned}
$$

so that for $n=0$,

$$
d C^{(0)} / d \Theta=-J
$$

and for $n=1$,

$$
\begin{aligned}
d C^{(1)} / d \theta= & -\exp [-(h+\epsilon) / \Theta] w(1 / \Theta-1) C^{(\lambda-1 / 3)} \\
& -J \xi^{*} .
\end{aligned}
$$

In our earlier isothermal work $^{6}$ we had $\omega=w(1 / \Theta-1)$ $\times \exp [-(h+\epsilon) / \Theta]$ defined as a constant. Equation (3.5) thus represents the temperature effect for asymptotic ripening according to the present theory. The temperature dependence of the denucleation term, $-J \xi^{*}$, is inherent in the computations illustrated below.

The exponential temperature dependence in Eq. (3.5) was proposed previously ${ }^{2,27}$ to describe ripening kinetics of $\mathrm{Ni}$ alloys, except that only the activation energy was in the exponent. According to the present theory, $h$ appears in addition to $\epsilon$ because of the microscopic reversibility implied by Eq. (2.6) along with Eq. (2.12). If only the forward rate constant is considered, as in the usual approach to Ostwald ripening, only the activation energy $\epsilon$ emerges in the exponent.

Now we assume $e^{6,14}$ that for long time, $C(\xi, \theta)$ has a scaled solution,

$$
C(\xi, \theta)=\theta^{-2 b} F\left(\left(\xi-\xi^{*}\right) \theta^{\chi}\right),
$$

which is a general form for the exponential solution found previously, ${ }^{4}$

$$
C(\xi, \theta)=\left[C^{(0)}(\theta / \beta(\theta)] \exp \left[-\left(\xi-\xi^{*}\right) / \beta(\theta)\right] .\right.
$$

Asymptotic ripening occurs ${ }^{4-6,14}$ such that

$$
C^{(0)}(\theta)=a_{0} \theta^{-b}
$$

and

$$
\beta(\theta)=C^{\mathrm{avg}}(\theta)-\xi^{*}=a_{1} \theta^{b} .
$$



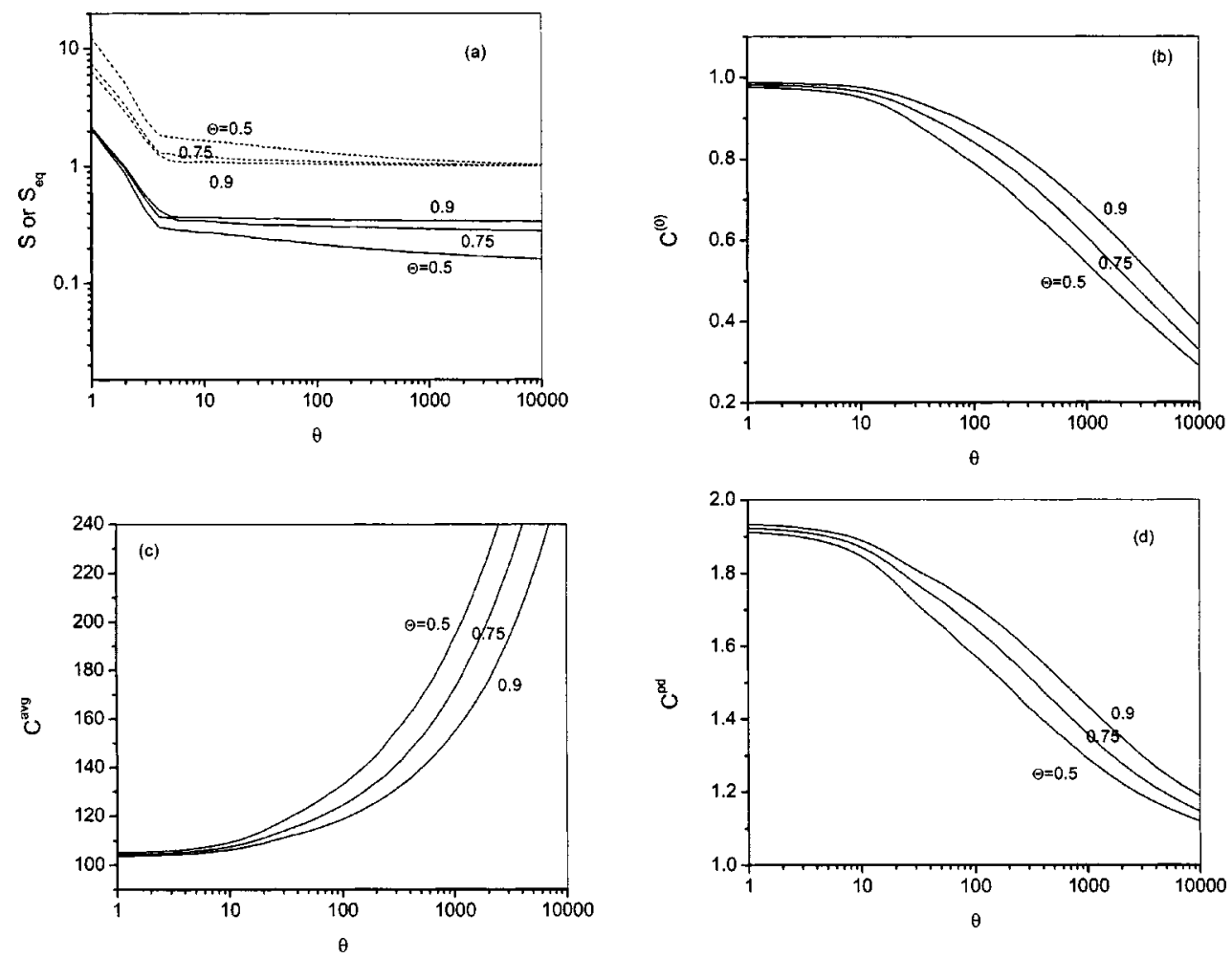

FIG. 1. Effect of scaled temperature $\Theta$ on the time evolution of (a) $S$ (solid line) and supersaturation ratio $S_{\text {eq }}$ (dashed line), (b) particle number concentration, $C^{(0)}$, (c) particle average mass $C^{\mathrm{avg}}$, (d) polydispersity $C^{\mathrm{pd}}$. The parameters in the calculations are $S_{0}=5, w=1, C_{0}^{(0)}=1, C_{0}^{\mathrm{avg}}=100, \lambda=0, \epsilon=0.01, h=1$.

Combining Eqs. (3.8) and (3.9) shows that the mass, $C^{(1)}$ $=C^{(0)} C^{\text {avg }}$, is asymptotically constant with $\theta$ and therefore

$$
\xi^{*}(\theta) \sim \theta^{b}
$$

As time increases, $S$ approaches its limiting value, so that the total cluster mass indeed becomes constant. The asymptotic solution, Eq. (3.7), is thus

$$
C(\xi, \theta)=\left[\theta^{-2 b} a_{0} / a_{1}\right] \exp \left[-\left(\xi-\xi^{*}\right) \theta^{-b} / a_{1}\right]
$$

where in Eq. (3.6), $\chi=-b$.

The moments of the exponential solution, Eq. (3.7), can be written for integer values of $n$,

$$
C^{(n)}(\theta)=C^{(0)}(\theta) \sum_{j=0}^{n}\left(\begin{array}{l}
n \\
j
\end{array}\right) \xi^{* n-j} \beta^{j} j !
$$

Substituting Eqs. (3.8)-(3.10) yields the asymptotic time dependence, $C^{(n)}(\theta) \sim \theta^{-b} \theta^{b n}$. With $d C^{(1)} / d \theta=0$ in Eq. (3.5), substituting Eqs. (3.5) and (3.8) along with Eq. (3.10) for $J \xi^{*}$ yields $\theta^{-b} \theta^{b(\lambda-1 / 3)} \sim \theta^{(-b-1)} \theta^{b}$, or

$$
b=1 /(4 / 3-\lambda),
$$

a result reported previously. ${ }^{6}$ This result indicates that the asymptotic, power-law time dependence of the particle size is independent of temperature and that only the rate of approach to this asymptote is influenced by temperature.

\section{NUMERICAL SOLUTION}

We consider an initial exponential distribution with smallest cluster mass, $\xi_{0}^{*}$,

$$
C_{0}(\xi)=\left[C_{0}^{(0)} / \beta_{0}\right] \exp \left[-\left(\xi-\xi_{0}^{*}\right) / \beta_{0}\right]
$$

which has the moments [Eq. (2.20)],

$$
C_{0}^{(n)}=C_{0}^{(0)} \sum_{j=0}^{n}\left(\begin{array}{c}
n \\
j
\end{array}\right) \xi_{0}^{* n-j} \beta_{0}^{j} .
$$

Thus, $C_{0}^{\mathrm{avg}}=\beta_{0}+\xi_{0}^{*}$ and $C_{0}^{\mathrm{var}}=\beta_{0}^{2}$. We choose the dimensionless zeroth moment $C_{0}^{(0)}=1$ and the ratio $S_{0}=5$. The initial average particle mass is $C_{0}^{\mathrm{avg}}=100$ and the polydispersity, $C_{0}^{\mathrm{pd}}$, defined as $C_{0}^{(2)} C_{0}^{(0)} / C_{0}^{(1) 2}$, is 2 for the exponential distribution, Eq. (4.2).

In our analysis, we show how the parameters $\Theta, h, \epsilon$, and $w$ (respectively, temperature, transition heat, activation energy, and interfacial energy) affect the evolution of the particle size distribution. The values of these parameters are chosen to be consistent with experimental values. The absolute temperature is scaled by a reference temperature, which for gas-liquid systems is the critical temperature $T_{c}$, so that the reduced temperature obeys $0<\Theta<1$. The molar energy of the phase transition, $\Delta H$, similar to a heat of crystallization, is usually in the range $0-3 \mathrm{kcal} / \mathrm{mol},{ }^{28}$ therefore, we have chosen $h\left(=\Delta H / R T_{c}\right) \sim 1$. The scaled activation energy for diffusion, $\epsilon$, is usually smaller than the molar energy of phase transition, $h$, e.g., for the ripening of precipitated amorphous alumina gel. ${ }^{29}$ However, $\epsilon$ can be greater than $h$ for ripening of metallic grains. ${ }^{2}$ The striking effects of choosing $\epsilon$ equal to 0.01 or 1.0 are presented below. The values of $w$ can be directly calculated from the fundamental parameters given by Eq. (2.16), which for vapor-liquid systems $^{30,31}$ range from 2 (methanol at $350 \mathrm{~K}$ ) to 33 (mercury at $290 \mathrm{~K}$ ). For solids, $w$ would be smaller than these values and we have chosen values around $w=1$. For the 

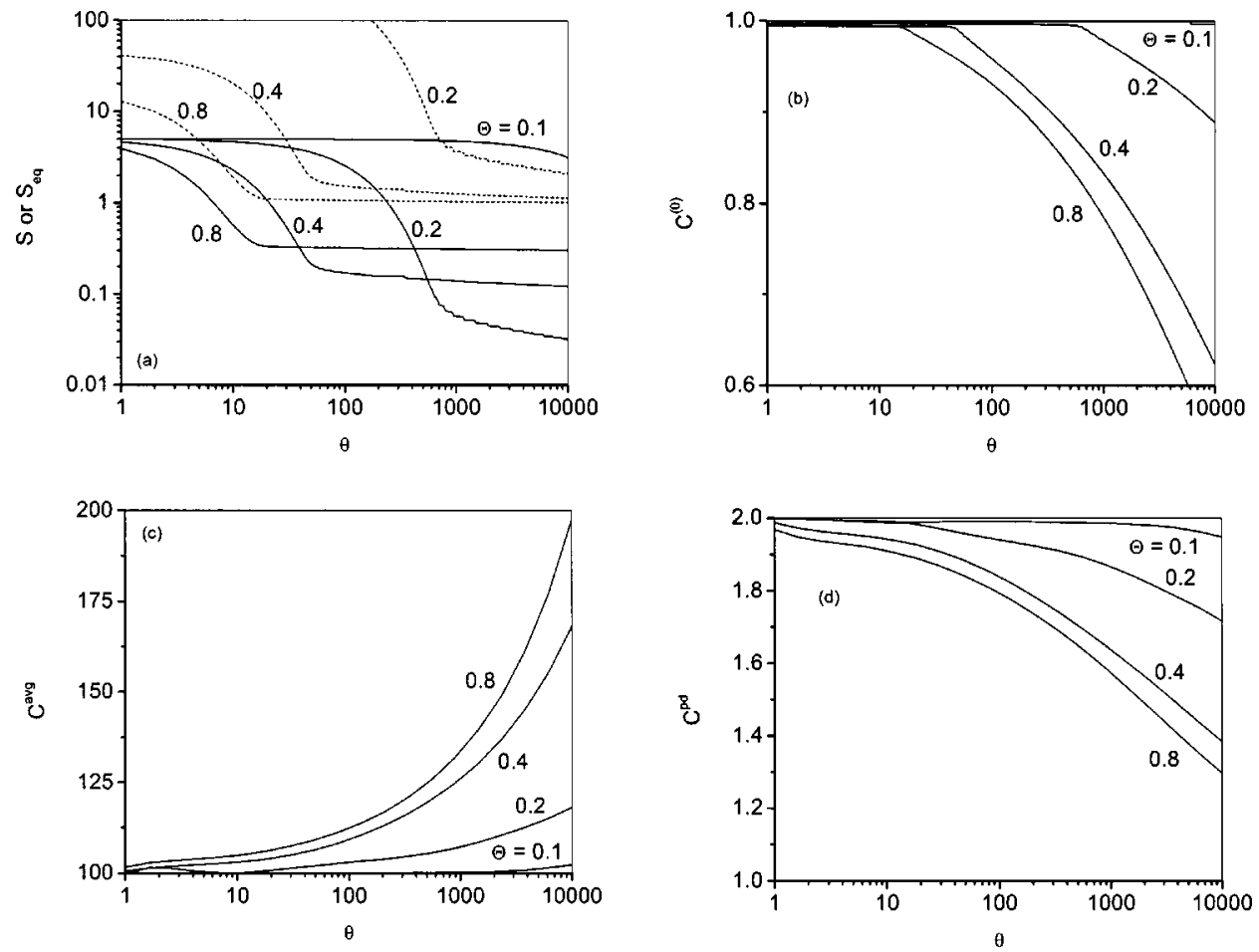

FIG. 2. Effect of scaled temperature $\Theta$ on the time evolution of (a) $S$ (solid line) and supersaturation ratio $S_{\text {eq }}$ (dashed line), (b) particle number concentration, $C^{(0)}$, (c) particle average mass $C^{\mathrm{avg}}$, (d) polydispersity $C^{\mathrm{pd}}$. The parameters in the calculations are $S_{0}=5, w=1, C_{0}^{(0)}=1, C_{0}^{\text {avg }}=100, \lambda=0, \epsilon=h, h=1$.

computational results presented below, the reduced time $\theta$ varies from 0.1 to 10000 , i.e., over five orders of magnitude. This explains the reasoning behind the parameter values chosen in this study.
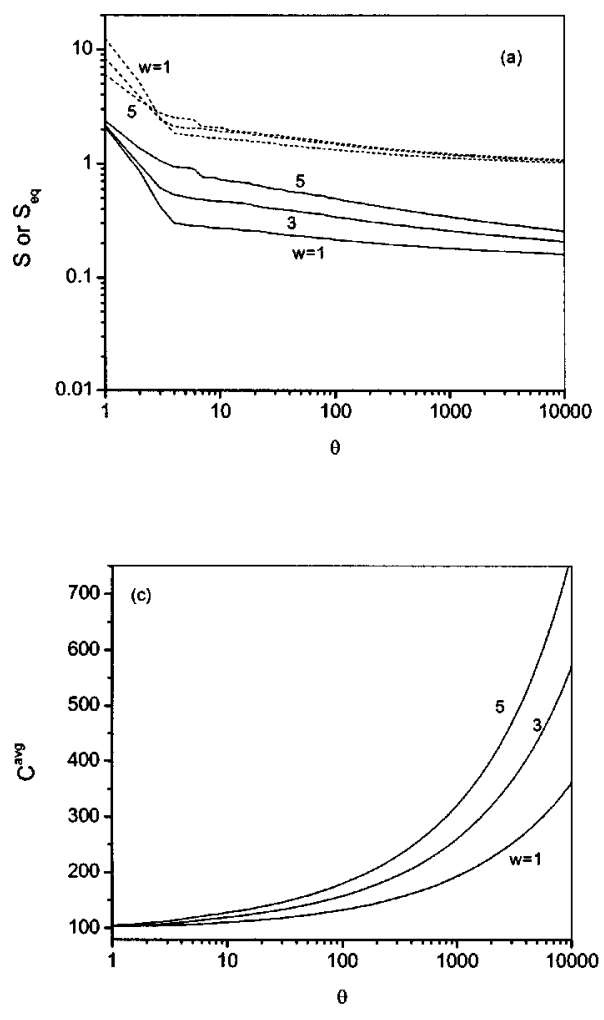

Equations (2.14) and (2.15) are simultaneous differential equations that were solved by a Runge-Kutta technique with an adaptive time step. ${ }^{32}$ The distribution $C(\xi, \theta)$ was evaluated at each time step sequentially. The mass variable $(\xi)$ was
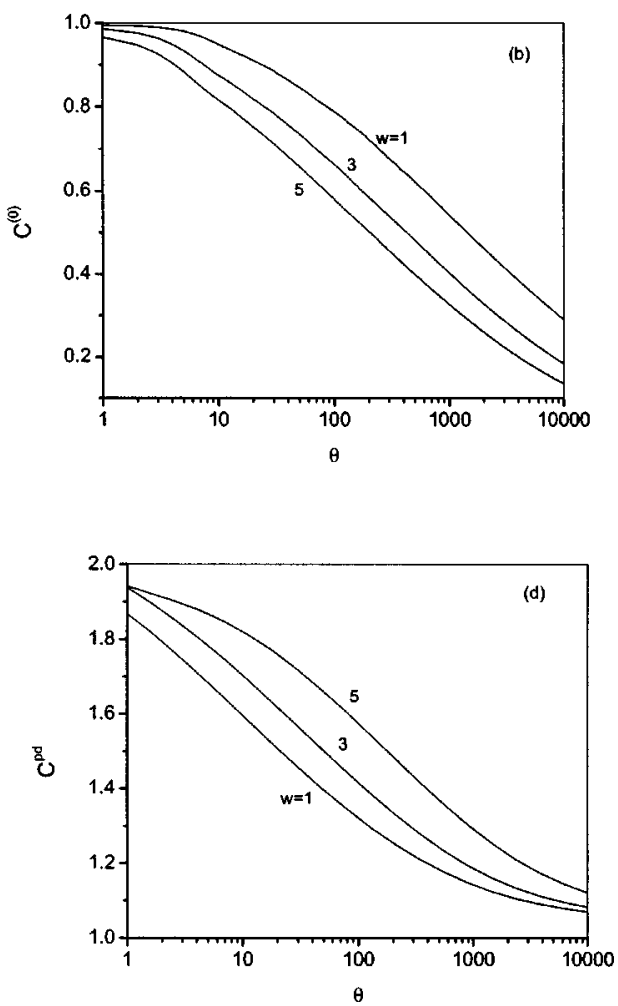

FIG. 3. Effect of interfacial energy $w$ on the time evolution of (a) $S$ (solid line) and supersaturation ratio $S_{\text {eq }}$ (dashed line), (b) particle number concentration, $C^{(0)}$, (c) particle average mass $C^{\text {avg }}$, (d) polydispersity $C^{\text {pd }}$. The parameters in the calculations are $S_{0}=5, \Theta=0.5, C_{0}^{(0)}=1, C_{0}^{\text {avg }}=100, \lambda=0, \epsilon=0.01$, $h=1$. 

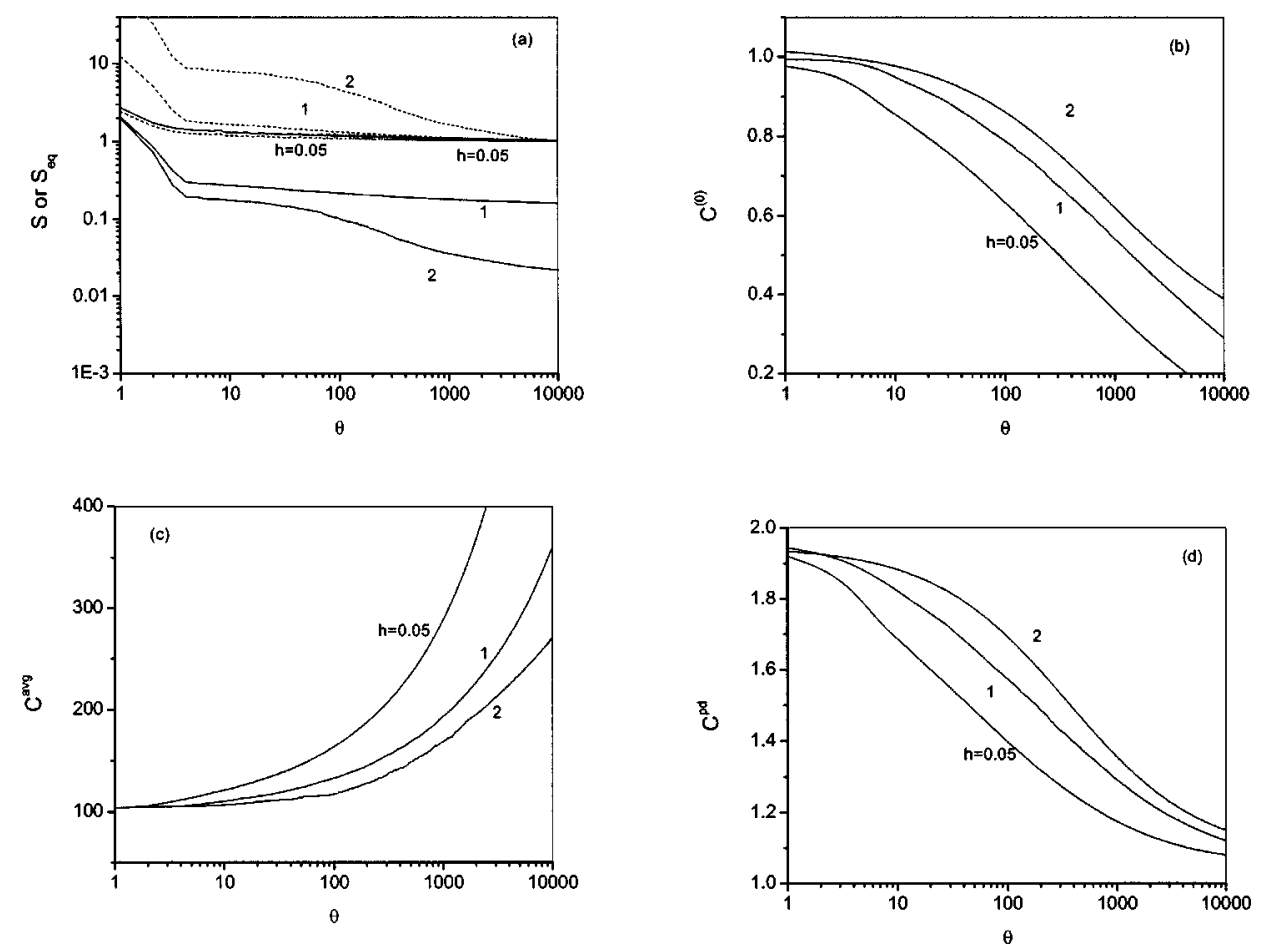

FIG. 4. Effect of scaled heat of conduction, $h$, on the time evolution of (a) $S$ (solid line) and supersaturation ratio $S_{\text {eq }}$ (dashed line), (b) particle number concentration, $C^{(0)}$, (c) particle average mass $C^{\text {avg }}$, (d) polydispersity $C^{\text {pd }}$. The parameters in the calculations are $S_{0}=5, \Theta=0.5, C_{0}^{(0)}=1, C_{0}^{\text {avg }}=100, \lambda=0$, $\epsilon=0.01, w=1$.

divided into 5000 intervals and the adaptive time $(\theta)$ step varied from 0.001 to 0.1 . The mass balance is confirmed at every step by comparing $S$ from the computation to $S$ [Eq. (2.15)] from the mass balance, Eq. (2.18). If the two values
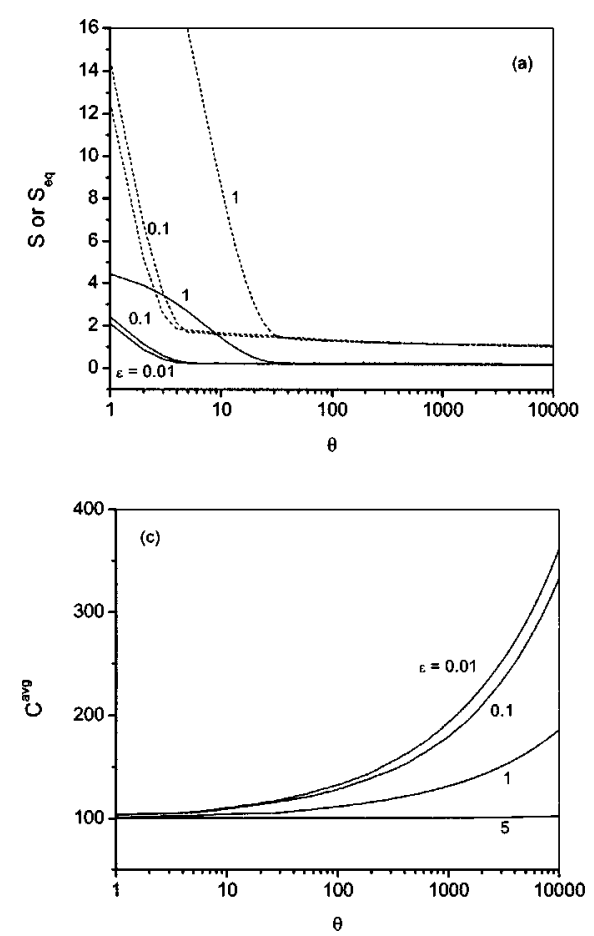

are within the prescribed tolerance of 0.01 , the iteration is permitted to continue. Several kinds of computations demonstrate how the model can be applied. Figure 1 shows, when $\epsilon=0.01$, the effect of $\Theta=\left(T / T_{c}\right)$ on the time evolution of
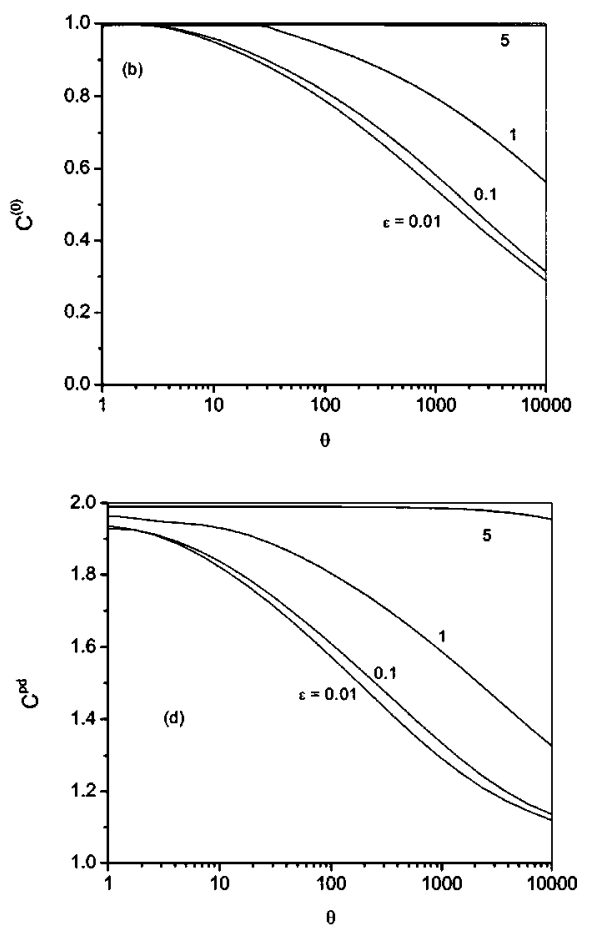

FIG. 5. Effect of scaled activation energy, $\epsilon$, on the time evolution of (a) $S$ (solid line) and supersaturation ratio $S_{\text {eq }}$ (dashed line), (b) particle number concentration, $C^{(0)}$, (c) particle average mass $C^{\text {avg }}$, (d) polydispersity $C^{\text {pd }}$. The parameters in the calculations are $S_{0}=5, \Theta=0.5, C_{0}^{(0)}=1, C_{0}^{\text {avg }}=100, \lambda=0$, $h=1, w=1$. 

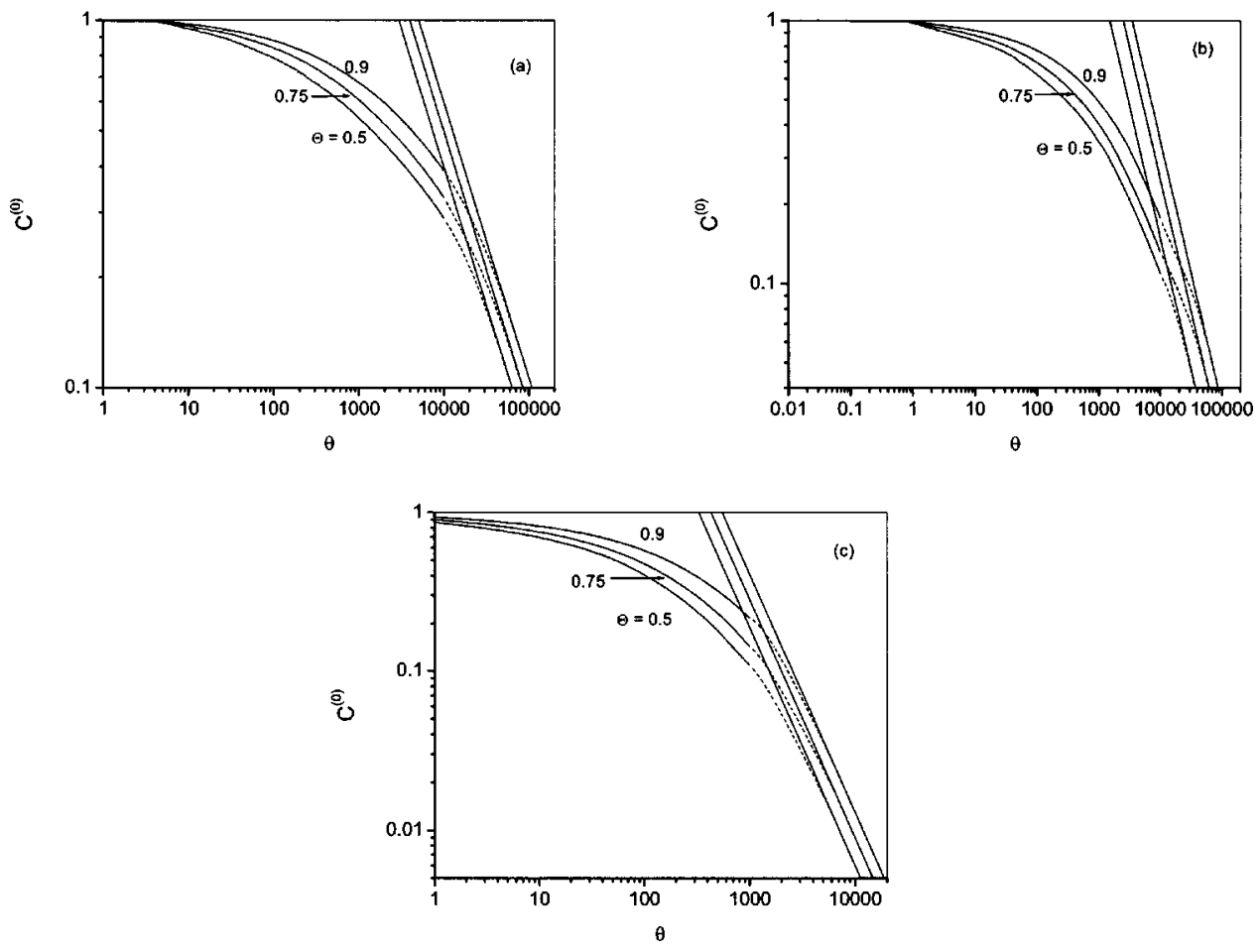

FIG. 6. Effect of scaled temperature $\Theta$ on the time evolution of the particle number concentration $C^{(0)}$ for (a) $\lambda=0$, (b) $\lambda=1 / 3$, (c) $\lambda=2 / 3$. The parameters in the calculations are $S_{0}=5, w=1, C_{0}^{(0)}=1, C_{0}^{\text {avg }}=100, \epsilon=0.01$, and $h=1$. As mentioned in the text, realistic units for $C^{(0)}$ and for $\theta$ are $10^{24}$ particles $/ \mathrm{m}^{3}$ and $10^{-7} \mathrm{~s}$.

the number concentration, average mass, supersaturation, and polydispersity. As shown in Fig. 1, the supersaturation decreases and average mass increases. The ratio $S$ $=m^{(0)} / \mu_{\infty}$ does not decrease to unity, but the temperaturedependent supersaturation ratio, $S_{\text {eq }}$, defined in Eq. (2.13), reaches unity, as shown in Fig. 1(a). The scaled particle number density, $C^{(0)}$, and the average mass, $C^{\text {avg }}$ decrease and increase, respectively, as expected during ripening. The polydispersity, $C^{\mathrm{pd}}$, evolves to unity as the distribution approaches a single particle after a very long time.

When $\epsilon$ is changed from its value of 0.01 in Fig. 1 to 1.0 in Fig. 2, the results reveal a reversal of temperature dependence of ripening rates. Figures 1(b) and 1(c) show that increasing the temperature decreases the ripening rate; both the number of particles and their average size evolve more slowly. Figures 2(b) and 2(c) show that increasing $T$ (or $\theta$ ) has the opposite behavior. This can be understood by recognizing that Eq. (2.15) with Eq. (2.16) shows how $J$ is directly influenced by $\exp (-\epsilon / \Theta)$ in the range when $S$ is changing only slowly. The denucleation rate $J$ is thus smaller for larger $\epsilon$ and vice versa, as displayed in Figs. 1 and 2. As expected, the larger activation energy has a greater effect on the ripening rate, actually reversing the weaker temperature dependence of smaller $\epsilon$.

Figure 3 shows the effect of the interfacial energy parameter $w$ on the time evolution of the number concentration, average mass, supersaturation, and polydispersity. The plots indicate that interfacial energies play an important role in determining the evolution of the distribution. The effect of molar energy of the phase transition, $h$, on the evolution of the crystal size distribution was investigated. Figure 4 shows that $h$ also can play a role in determining the time evolution of particle distribution.

Figure 5, consistent with Figs. 1 and 2, shows the effect of the activation-energy parameter $\epsilon$ on the time evolution of the crystal size distribution. The parameter is varied three orders of magnitude and it is evident that ripening decreases as $\epsilon$ increases, owing to the smaller rate coefficients [Eqs. (2.8) and (2.6)].

The central issue of Ostwald ripening is the asymptotic time dependence of the particle number density (or average particle size). Figure 6 shows the evolution of the number density for different values of $\lambda$, the power on cluster mass [Eq. (2.6)] for the rate coefficients. The value $\lambda=0$ signifies mass-independent rate coefficients, $\lambda=1 / 3$ represents diffusion-controlled ripening, and $\lambda=2 / 3$ represents surfacecontrolled ripening. Our numerical method allows computations up to when the slopes on the log-log graphs are approaching their temperature-independent asymptotes. ${ }^{6}$ The dashed lines in Fig. 6 are extrapolated to the asymptotic slope $1 /(4 / 3-\lambda)$, derived as Eq. (3.13). The experimental data ${ }^{33}$ for coarsening of spherical $\mathrm{Ni}_{3} \mathrm{Al}$ precipitates in a $\mathrm{Ni}-\mathrm{Al}$ alloy has asymptotic slope -0.73 and corresponds to Fig. 6(a) with $\Theta=0.75$ if we specify $C^{(0)}$ in units of $10^{24}$ particles $/ \mathrm{m}^{3}$, time in units of $10^{-7} \mathrm{~s}$, and temperature in units of $1364 \mathrm{~K}$.

We show how linear temperature programming can control crystal or grain growth processes in Fig. 7, where temperature decreases with time. Ostwald ripening increases with decreasing temperature such that average size increases while polydispersity decreases. Such a strategy of temperature programming provides a technique to control the growth 

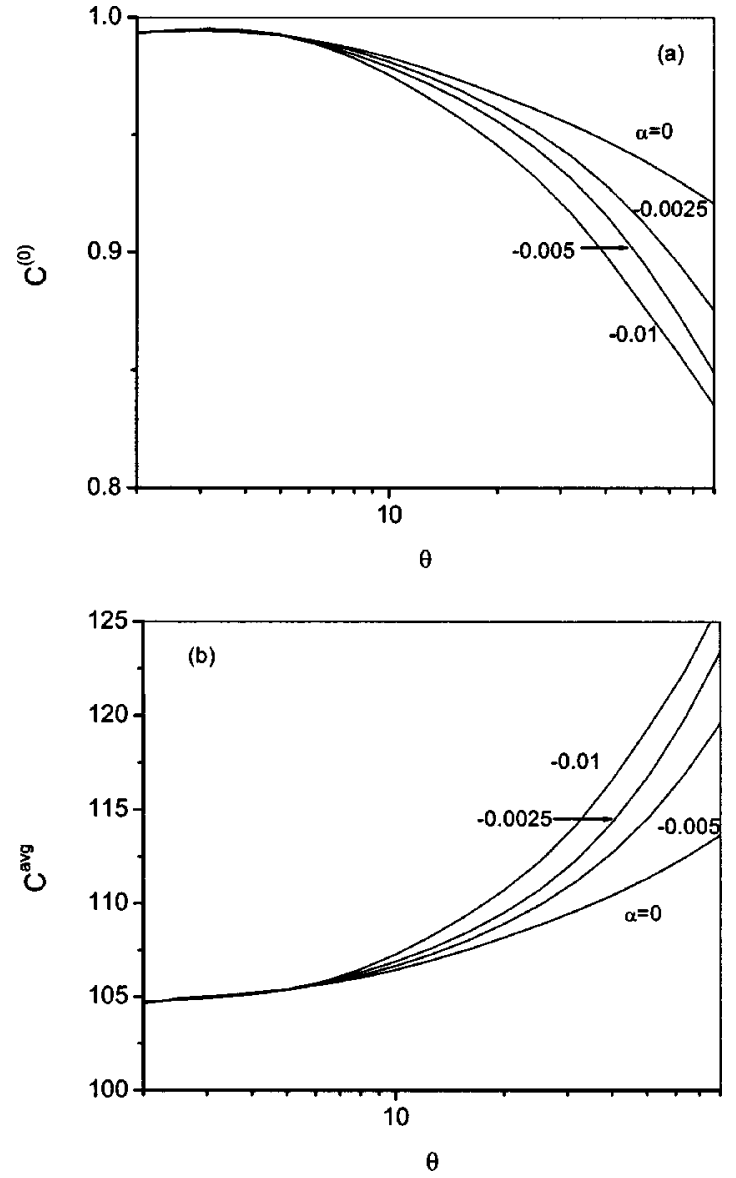

FIG. 7. Effect of the linear temperature-rate parameter $\alpha$ on the time evolution of (a) particle number concentration $C^{(0)}$, and (b) particle average mass $C^{\text {avg }}$. The parameters in the calculations are $\Theta=0.95+\alpha \theta, C_{0}^{(0)}=1$, $C_{0}^{\mathrm{avg}}=100, S_{0}=5, w=1, \lambda=0, \epsilon=0.01, h=1$.

of crystals. As temperature decreases, the liquid solution may eventually freeze, immobilizing the grains in a solid matrix.

Because $\theta$ varies between 0 and 1, a large linear temperature rate of change is applicable only for short times. Therefore, an assumed exponential temperature program given by $\Theta=0.05+0.9(\exp (-\alpha \theta))$ allows $\Theta$ to vary from an initial value of 0.95 to a final value of 0.05 . Figure 8 shows the variation of concentration, average mass, supersaturation, and polydispersity for $\alpha=0.001$ and 0.01 .

We show the time evolution of two polymorphs with increasing temperature. Polymorphs have different properties, including solubility ${ }^{18}$ and interfacial effects. ${ }^{21}$ Polymorphs of different shape may have larger differences in $w$ than in $h$, which can have an important effect in determining the evolution of crystal size distributions. We hypothesize that differences in the time evolution of the polymorphs are influenced by the interfacial energies, $w_{\mathrm{A}}$ and $w_{\mathrm{B}}$, rather than activation or phase transition energies. Thus, we examine two distributions, $C_{\mathrm{A}}(\xi, \theta)$ and $C_{\mathrm{B}}(\xi, \theta)$, which have moments defined in Eq. (2.15) appropriately subscripted. The same solute produces the two polymorphs. The differential equations (2.20)-(2.22) are solved by the technique described previously, ensuring the mass balance, Eq. (2.19), is satisfied.

Figure 9 demonstrates how two polymorphs with differ- ent interfacial energies, $w_{\mathrm{A}}$ and $w_{\mathrm{B}}$, but the same initial number density and average size, would evolve with time during temperature programming. As shown in Fig. 9, the A polymorph has the lower interfacial energy and grows in mass and energy size while the number and mass concentrations of the B polymorph decrease to zero. Thus, the B form nearly disappears while the A form ripens to a larger size.

\section{DISCUSSION AND CONCLUSIONS}

The lack of a suitable theory has meant that the implications of ripening and its temperature dependence might be difficult to recognize. A recent discussion of grain nucleation and growth during phase transformation of carbon steel ${ }^{34}$ illustrates the point. The work was based on a novel experimental approach by $\mathrm{x}$-ray diffraction at a synchrotron source. ${ }^{35}$ Observations of decreasing number of grains during cooling were explained by postulating a decreased nucleation rate below that predicted by classical nucleation theory. The critical nucleus of $10-100$ atoms $^{29}$ was considerably smaller than the smallest detectable grain diameter of $4 \mu \mathrm{m}$. This suggests that instead of monitoring the nucleation rate, the experiment actually measured the number of grown and ripened grains. It is clear from Fig. 7 that cooling during ripening can enhance the rate of denucleation, thus possibly accounting for the decreased number of grains observed. Although polycrystalline phase transformations in steel are extremely complex involving several alloying elements and several solid-state phase transformations, the present theory of ripening suggests an alternative explanation for the experimental observations. The imposed cooling enhances grain loss due to Ostwald ripening, thus simulating the experimental observations. Such an elucidation based on classical ideas of ripening allows one to avoid questioning classical nucleation theory.

Metallurgical materials are subjected to annealing heat treatments to improve their mechanical properties, including strength and toughness, which depend upon grain composition, shape, and size distribution. The final state of the alloy is a complex function of how nucleation, growth, and ripening occur during the heat treatment. In practice, deformation processes also influence the metallic state. A recent study ${ }^{36}$ of heat treatment of two-phase titanium alloys found that aging at higher temperatures led to ultra-fine silicides, consistent with Fig. 1(c), which shows smaller grains after ripening at higher temperatures for a given time. The observed complex effect ${ }^{36}$ of temperature programming is reflected in Fig. 8, where lines for average grain size cross. Further evidence is provided by a study ${ }^{37}$ of precipitation strengthening of $\mathrm{Al}(\mathrm{Sc})$ alloys at different temperatures. When alloys aged at $300{ }^{\circ} \mathrm{C}$ and $400{ }^{\circ} \mathrm{C}$ are compared, the higher temperature yields the larger size precipitates, in agreement with Fig. 2 (c). This suggests a relatively large activation energy in this system.

We have attempted in our work on phase-transition dynamics to develop a consistent and comprehensive theory for particle (crystal or droplet) growth and ripening. The aim is to establish guidelines for including the essential elements that must appear in a realistic model. The proposed theory, 

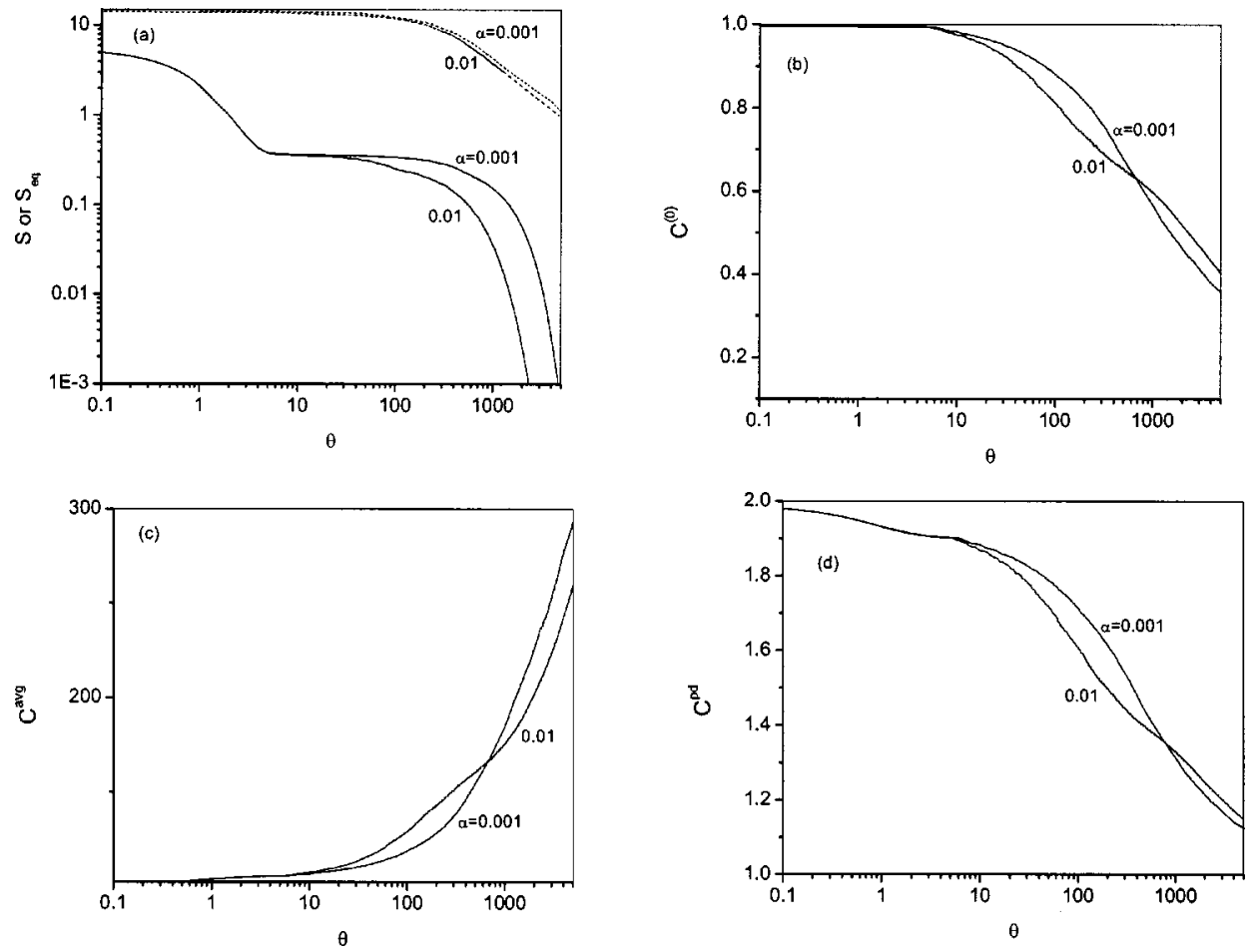

FIG. 8. Effect of exponential temperature-rate parameter $\alpha$ on the time evolution of (a) $S$ (solid line) and supersaturation ratio $S_{\text {eq }}$ (dotted line), (b) particle number concentration $C^{(0)}$, (c) particle average mass $C^{\text {avg }}$, (d) polydispersity $C^{\text {pd }}$. The parameters in the calculations are $\Theta=0.5+0.9(\exp (-\alpha \theta))$, $C_{0}^{(0)}=1, C_{0}^{\mathrm{avg}}=100, S_{0}=5, w=1, \lambda=0, \epsilon=0.01, h=1$.

based on distribution kinetics, provides a way to understand and compute how the particle-size distribution and its properties develop in time. Such a theory is useful in understanding observed behavior during phase transitions and in plan- ning effective and efficient processes for manufacturing materials. The current article has explored the temperature effects on the last stage of condensation, Ostwald ripening. The proposed model allows key physical properties (interfa-
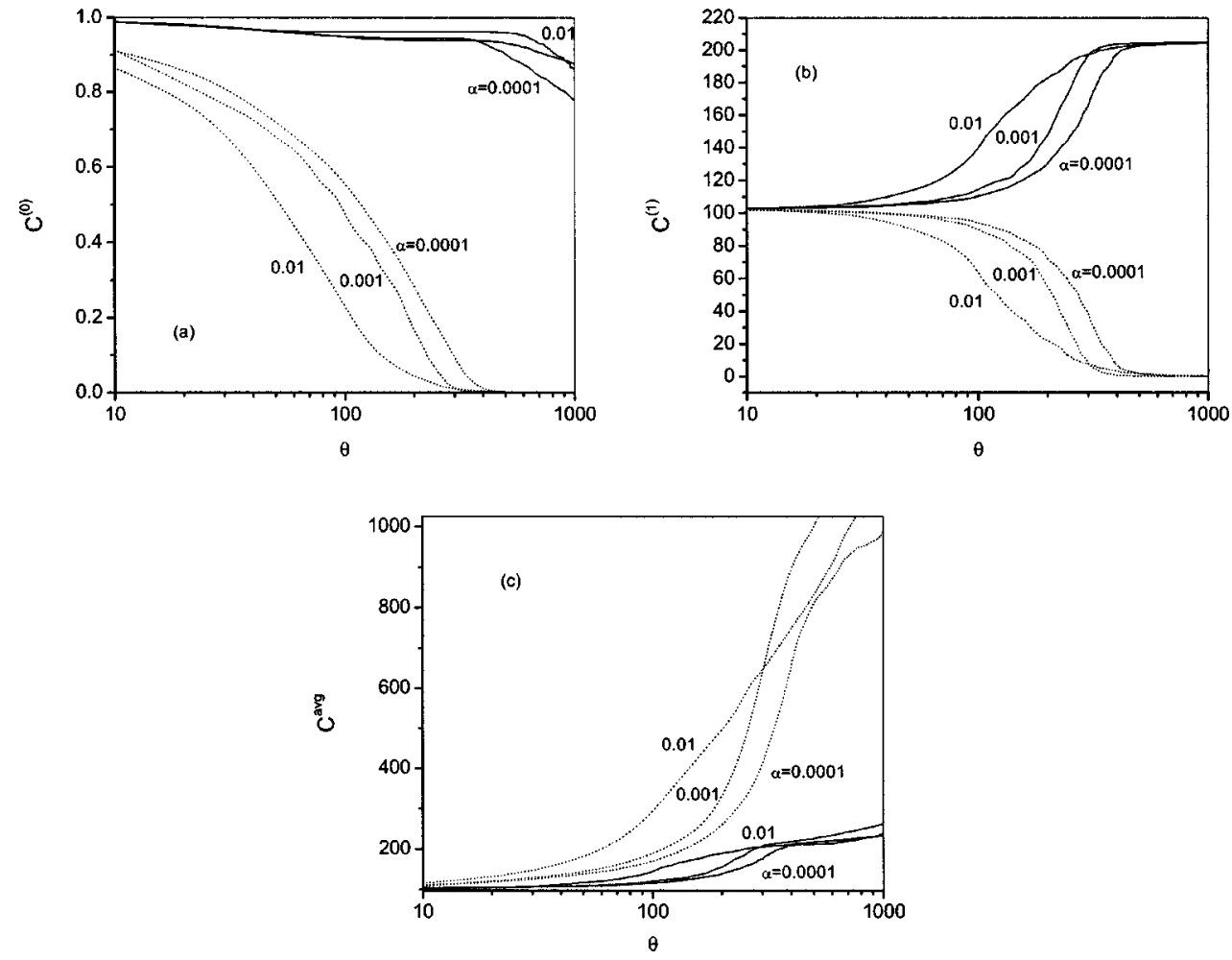

FIG. 9. Evolution of two polymorphs, A (solid line) and B (dotted line), as a function of the temperature-rate parameter $\alpha$ for (a) particle number concentrations, (b) particle mass concentrations, and (c) particle average masses. The parameters in the calculations are $\theta=0.05+0.9(\exp (-\alpha \theta))$, $C_{\mathrm{A} 0}^{(0)}=C_{\mathrm{B} 0}^{(0)}=1, C_{\mathrm{A} 0}^{\mathrm{avg}}=C_{\mathrm{B} 0}^{\mathrm{avg}}=100, w_{\mathrm{A}}=1, w_{\mathrm{B}}=2, S_{0}=5, \lambda=0, \epsilon=0.01, h=1$. 
cial energy, heat of condensation, activation energy, GibbsThomson curvature effect on growth rate and on the critical nucleus size) to be incorporated into the quantitative evaluation of an evolving size distribution. The distributionkinetics approach begins with the population dynamics equation that describes the dependence of the size-distribution functions on time and particle mass. The equation includes particle growth and dissolution kinetics, as well as the denucleation rate for particles that have shrunk to their critical nucleus size. An accompanying equation for the solution concentration affords a mass balance for particle mass and dissolved solute. The mass moments of the distribution, which is solved numerically in scaled (dimensionless) form, yield the particle number concentration (zeroth moment) and mass concentration (first moment), and hence average particle mass. As a measure of the distribution's width, the polydispersity index is based on the second mass moment. As in previous work we find asymptotic power-law temporal behavior for decreasing particle concentration and increasing average particle size.

With temperature as an active parameter in the model, one can determine not only the influence of different temperatures on ripening, but also the effect of temperature programming. Changing temperature with time can potentially control particulate size distributions based on realistic modeling of crystallization processes during cooling or heating. We have presented results for linear and for exponential temperature processes, and have demonstrated enhanced ripening during cooling. Temperature programs further provide a method to distinguish and possibly separate polymorphs (different structural forms with the same crystalline composition). If two polymorphs have different interfacial energies, which could cause different shapes for the two forms, then the computations suggest that ripening may manifest different dynamics, and hence separation. Increasing the temperature according to an exponential program demonstrates that the polymorph with larger interfacial energy will essentially dissolve away, while the polymorph with smaller energy increases in mass.
${ }^{1}$ A. Baldan, J. Mater. Sci. 37, 2171 (2002).

${ }^{2}$ A. Baldan, J. Mater. Sci. 37, 2379 (2002).

${ }^{3}$ P. W. Voorhees, Annu. Rev. Mater. Sci. 22, 197 (1992).

${ }^{4}$ G. Madras and B. J. McCoy, J. Chem. Phys. 115, 6699 (2001).

${ }^{5}$ G. Madras and B. J. McCoy, J. Chem. Phys. 117, 6607 (2002).

${ }^{6}$ G. Madras and B. J. McCoy, J. Chem. Phys. 117, 8042 (2002).

${ }^{7}$ I. M. Lifshitz and J. Slyozhov, J. Phys. Chem. Solids 19, 35 (1961).

${ }^{8}$ C. Wagner, Z. Elektrochem. 65, 243 (1961).

${ }^{9}$ J. A. Marqusee and J. Ross, J. Chem. Phys. 79, 373 (1983).

${ }^{10}$ G. Venzl, Ber. Bunsenges. Phys. Chem. 87, 318 (1983).

${ }^{11}$ A. Bhakta and E. Ruckenstein, J. Chem. Phys. 103, 7120 (1995).

${ }^{12}$ G. Madras and B. J. McCoy, Chem. Eng. Sci. (to be published).

${ }^{13}$ H. Gratz, J. Mater. Sci. Lett. 18, 1637 (1999).

${ }^{14}$ K. Binder, Phys. Rev. B 15, 4425 (1977).

${ }^{15}$ H. Xia and M. Zinke-Allmang, Physica A 261, 176 (1998).

${ }^{16}$ M. Zinke-Allmang, L. C. Feldman, and M. H. Grabow, Surf. Sci. Rep. 16, 377 (1992).

${ }^{17}$ G. R. Carlow and M. Zinke-Allmang, Surf. Sci. 328, 311 (1995).

${ }^{18}$ R. Mohan, K.-K. Koo, C. Strege, and A. S. Myerson, Ind. Eng. Chem. Res. 40, 6111 (2001).

${ }^{19}$ D. B. Patience and J. B. Rawlings, AIChE J. 47, 2125 (2001).

${ }^{20}$ V. A. Snyder, N. Akaiwa, J. Alkemper, and P. W. Voorhees, Metall. Mater. Trans. A 30A, 2341 (1999).

${ }^{21}$ G. Madras and B. J. McCoy, Acta. Mater. 51, 2031 (2003).

${ }^{22}$ B. J. McCoy, J. Colloid Interface Sci. 228, 64 (2000).

${ }^{23}$ G. Madras and B. J. McCoy, J. Cryst. Growth 243, 204 (2002).

${ }^{24}$ D. F. Calef and J. M. Deutch, Annu. Rev. Phys. Chem. 34, 493 (1983).

${ }^{25}$ M. Strobel, K.-H. Heinig, and W. Moller, Phys. Rev. B 64, 245422 (2001).

${ }^{26}$ R. C. Reid, J. M. Prausnitz, and T. K. Sherwood, The Properties of Gases and Liquids (McGraw-Hill, New York, 1977), p. 612.

${ }^{27}$ S. Olive, U. Grafe, and I. Steinbach, Comput. Mater. Sci. 7, 94 (1996).

${ }^{28}$ R. H. Perry and D. W. Green, Perry's Chemical Engineers' Handbook, 7th ed. (McGraw-Hill, New York, 1997), Table 2-224.

${ }^{29}$ J. M. Rousseaux, P. Weisbecker, H. Muhr, and E. Plasari, Ind. Eng. Chem. Res. 41, 6059 (2002).

${ }^{30}$ S. L. Girshick and C.-P. Chiu, J. Chem. Phys. 93, 1273 (1990).

${ }^{31}$ C. H. Yang and H. Liu, J. Chem. Phys. 84, 416 (1986).

${ }^{32}$ W. H. Press, S. A. Teukolsky, W. T. Vetterling, and B. P. Flannery, Numerical Recipes in C: The Art of Scientific Computing (Cambridge University Press, New York, 1993).

${ }^{33}$ A. J. Ardell, Mater. Sci. Eng. A238, 108 (1997).

${ }^{34}$ S. E. Offerman, N. H. van Dijk, J. Sietsma, S. Grigull, E. M. Lauridsen, L. Margulies, H. F. Poulsen, M. Th. Rekveldt, and S. van der Zwaag, Science 298, 1003 (2002).

${ }^{35}$ M. Militzer, Science 298, 975 (2002).

${ }^{36}$ X. D. Zhang, P. Bonniwell, H. L. Fraser, W. A. Baeslack, D. J. Evans, T. Ginter, T. Bayha, and B. Cornell, Mater. Sci. Eng., A 343, 210 (2003).

${ }^{37}$ D. N. Seidman, E. A. Marquis, and D. C. Dunand, Acta Mater. 50, 4121 (2002). 